\title{
Manufatura aditiva por estereolitografia: análise da geometria da peça e da influência da posição e orientação de fabricação
}

\author{
Additive manufacturing by stereolithography: \\ analysis of the part geometry and influence \\ of manufacturing position and orientation
}

\author{
Arthur Wilson Fonseca Coelho ${ }^{1,2}$, Anna Carla Araujo ${ }^{1}$,
} Rossana Mara da Silva Moreira Thiré ${ }^{2}$

\footnotetext{
${ }^{1}$ Centro de Estudos em Fabricação e Comando Numérico, Programa de Engenharia Mecânica, PEM/COPPE/UFRJ, CP: 68505, CEP: 21941-972, Rio de Janeiro, Rio de Janeiro, Brasil.

${ }^{2}$ Laboratório de Biopolímeros e de Bioengenharia, Programa de Engenharia Metalúrgica e de Materiais, PEMM/COPPE/UFRJ, Rio de Janeiro, Rio de Janeiro, Brasil.

e-mail: awf.coelho@mecanica.ufrj.br, anna@ufrj.br, rossana@metalmat.ufrj.br
}

\begin{abstract}
RESUMO
A estereolitografia é um dos processos de manufatura aditiva mais utilizados e utiliza resina líquida fotopolimerizável como matéria-prima. Por meio de uma reação de cura, cada camada desta resina é transformada para o estado sólido. Como qualquer tipo de processo de fabricação, a estereolitografia tem suas limitações e dificuldades, a mais comum é a contração dimensional das peças fabricadas durante a polimerização. É possível reduzir este defeito analisando os parâmetros que influenciam o fator de contração das peças produzidas, porém não foram encontrados estudos científicos que quantifiquem a influência da orientação da peça e se há influência da posição na plataforma. Neste artigo, a contração de peças fabricadas foi quantificada e a análise de variância dos resultados em diferentes orientações e localizações foi realizada. De acordo com os resultados, verificou-se que a localização das peças na plataforma é um fator importante em função das orientações das dimensões das geometrias fabricadas e portanto deve ser compensado de forma não homogênea para corrigir este defeito.
\end{abstract}

Palavras-chave: Impressão 3D, SLA, Qualidade dimensional, Contração, ANOVA.

\begin{abstract}
Stereolithography (SLA) is one of the most used processes of additive manufacturing and it uses a liquid resin as raw material. Based on a curing reaction each layer of this resin turns into a solid part. As any kind of manufacturing process, it has its limitations and difficulties, shrinkage is the common one that occurs during photopolymerization. It is possible to minimize it analyzing the parameters that affects the shrinkage factor, however, it was not found studies that analyze the influence of the orientation and position on the platform. In this article, the shrinkage of parts is measured and an analysis of variance of different oriented parts and positions is performed. According to the results, it was found that the location of the parts on the platform is an important factor according to the orientations of the manufactured geometries dimensions, so it should be considered as non-homogeneous during shrinkage correction.
\end{abstract}

Keywords: 3D Printing, SLA, Dimensional Quality, Shrinkage, ANOVA.

\section{INTRODUÇÃO}

Manufatura aditiva (Additive Manufacturing - AM) é um processo de fabricação em que camadas de pequena espessura são construídas uma sobre as outras para a formação de uma peça sólida. Esse tipo de processo de fabricação foi introduzido pela primeira vez em 1986 com o advento do processo de Estereolitografia (Stereolithography - SLA) por meio da patente de Charles Hull [1]. Atualmente, a AM já é utilizada em 
diversas áreas como a área automotiva [2], aeroespacial [3] e médica [4,5], sendo a estereolitografia um dos processos de AM mais utilizados nos dias de hoje [6]. Essa técnica tem o potencial de ser útil em diversas aplicações como, por exemplo, na área de microfluídica [7], Sistemas MicroEletroMecânicos (Micro Electrical Mechanical Systems - MEMS) [8], estruturas porosas para tecidos vivos [9,10] e moldes [11].

A SLA possui duas técnicas de formação da camada 2D distintas: Estereolitografia por Escaneamento (Scanning based Stereolithography - SSL) e Estereolitografia por Projeção (Projection-based Stereolithography - PSL) [12]. No processo de projeção se usa uma radiação ultravioleta (UV) que é projetada sobre toda a área da camada 2D, como apresenta a Fig. 1. O equipamento utilizado neste processo consiste de um emissor de radiação UV, uma lente pela qual a radiação converge e atinge um chip conhecido como Dispositivo Microespelhado Digital (DMD - Digital Micromirror Device), que tem a função de formar a imagem de cada camada da peça produzida. A imagem formada passa pela lente de projeção do projetor e então atinge um espelho que está posicionado a $45^{\circ} \mathrm{em}$ relação ao projetor e ao reservatório. A radiação ao atingir a camada de resina entre a plataforma e o fundo do reservatório faz com que a resina fotopolimerize, realizando o processo de cura, ou seja, formando uma camada de polímero sólido [12,13].

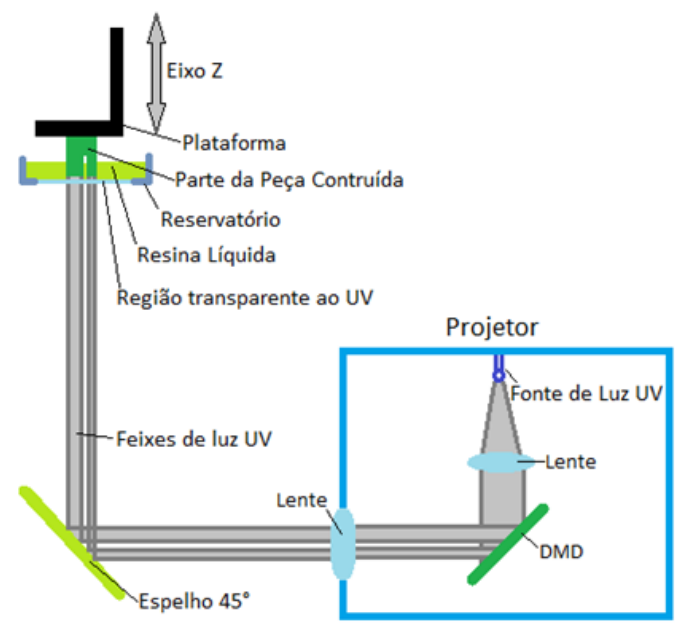

Figura 1: Processo de SLA por projeção (PSL) por exposição de fundo.

Comparado a outros processos de manufatura aditiva, o processo de estereolitografia consegue obter grande precisão e acurácia [13]. A alta resolução da projeção do laser ou da radiação UV e ao reduzido tamanho de camada proporciona esta qualidade dimensional. No entanto, diversas imperfeições podem ser encontradas nas peças produzidas por SLA, pois ainda há limitações que podem influenciar na peça fabricada, das quais a mais crítica é a contração dimensional.

O fenômeno de contração acontece tanto durante a fase de construção da peça quando na fase de póscura, quando o laser ou radiação UV fotopolimeriza a resina. A transformação dos monômeros (no estado líquido) em um polímero de ligação cruzada (estado sólido) faz com que haja o crescimento do número de ligações covalentes, que por serem mais fortes que as forças intermoleculares de Van der Waals, promovem contração da área da camada [14]. Assim, a contração está diretamente relacionada ao grau de polimerização da resina e, consequentemente, aos fatores que o influenciam diretamente, por exemplo, a concentração de fotoiniciadores, a intensidade da radiação, a temperatura de cura e a estrutura do monômero [15].

No artigo de ZHOU et al. [16], a precisão dimensional e geométrica de peças produzidas por SLA por escaneamento (SSL) foi avaliada em função da influência da espessura de camada, da distância entre as trajetórias do laser em cada camada, a influência do grau de sobre-cura, da distância entre o espalhador e a superfície da resina e a posição na plataforma. Foi observado que o grau de sobre-cura, a distância entre caminhos do laser e a posição da peça na plataforma foram os fatores que mais influenciam a acurácia.

Para minimizar o efeito da contração em peças fabricadas por SLA, HUANG et al. [17] desenvolveram um método matemático para otimizar sua geometria. Por meio de dados experimentais, foi possível realizar a previsão das regiões da camada que são mais suscetíveis à contração em função da posição angular e radial. Desta forma, um termo em função de coordenadas polares foi adicionado à equação que descreve a forma da peça no plano $x y$. Com esta correção na equação, foi possível compensar o efeito da contração em geometrias com formas cilíndricas. Posteriormente, este método foi aprimorado para formas 
livres, onde a geometria pode ser aproximada por meio de polígonos ou seções circulares [18].

A partir da teoria clássica de laminados e por meio de medição experimental, Karalekas e Aggelopoulos [19] estudaram as deformações (warpage) causadas em peças produzidas por SSL devido ao fenômeno da contração de uma resina acrílica. As peças produzidas tiveram como pós-processo aplicação de radiação ultravioleta ou pós-cura por alta temperatura. Foi visto que o primeiro pós-processo foi responsável por causar uma contração uniforme, enquanto que o segundo fez com que a peça tivesse uma contração mais acentuada em especial na direção correspondente ao eixo $y$ do equipamento.

WANG et al. [15] realizaram um estudo sobre quais dos parâmetros do processo que podem influenciar na contração de peças fabricadas por SLA por escaneamento. Foram avaliados: a potência do laser UV, a espessura de cada camada, a distância entre cada passagem do laser em uma mesma camada, a velocidade de avanço do traçado do laser, a estabilidade do laser e a taxa de absorção de radiação da resina. No entanto, não foi estudado o efeito da contração sob a influência da posição e da orientação da peça.

Tendo em vista a escassez de trabalhos publicados sobre o efeito dos parâmetros de impressão nas propriedades de peças produzidas por SLA por projeção (PSL), no presente estudo, foi avaliada a influência da localização em função da orientação da peça na plataforma de impressão na contração dimensional das peças fabricadas. Foi utilizado o mesmo material para produzir todas as peças, assim como foi mantida a mesma espessura da camada e o grau de sobre-cura. As medidas das peças para a avaliação dimensional foram realizadas com um perfilômetro digital.

\section{MATERIAIS E MÉTODOS}

A realização dos experimentos foi composta de duas fases: a manufatura da peça teste, com geometria idealizada para a avaliação dos parâmetros de fabricação, e a avaliação dimensional. Em um estudo anterior, Coelho [13] realizou a avaliação de acurácia na espessura de aletas fabricadas pelo mesmo equipamento.

\subsection{Equipamento de SLA e Material Polimérico}

O equipamento utilizado neste estudo foi uma impressora SLA por projeção, Projet 1200 da 3D Systems, capaz de produzir peças sob uma plataforma com dimensões de $43 \mathrm{~mm}$ de comprimento e $27 \mathrm{~mm}$ de largura (nas direções de impressão $x$ e $y$, respectivamente). Estas dimensões representam a maior área de fabricação de cada camada com uma altura máxima de impressão de $150 \mathrm{~mm}$ (direção $z$ ). A resolução no plano $x y$ deste equipamento é de $0,056 \mathrm{~mm}$ e a altura da camada foi constante para os experimentos, com espessura de camada de $0,03 \mathrm{~mm}$. Neste equipamento, os únicos parâmetros de fabricação que podem ser modificados são: a localização da impressão na plataforma, a taxa de sobre-cura, o fator de compensação de contração e a orientação de construção das peças. A matéria-prima líquida contida no cartucho de impressão utilizada para fabricar o corpo de prova são: dois monômeros, trietileno glicol diacrilato e diacrilato de triciclodecano dimetanol, tendo como fotoiniciador o óxido de fenil-bis(2,4,6- trimetilbenzoil)-fosfina, fornecido pela $3 D$ Systems com o nome comercial VisiJet FTX Green.

\subsection{Parâmetros de Impressão e Planejamento de Experimentos}

Três peças (réplicas) de mesmas dimensões nominais foram fabricadas conforme Fig. 2. A geometria projetada para fazer a análise dimensional possui uma base de $43 \mathrm{~mm}$ de comprimento e $26 \mathrm{~mm}$ de largura, ocupando toda a área da plataforma de impressão.

O tempo da deposição de camadas para cada peça foi de 17 minutos. Após a deposição de camadas, todas as peças foram finalizadas com a aplicação de solvente, o álcool isopropílico foi utilizado para remoção da resina não curada. Após a segunda etapa, a última fase foi a pós-cura da peça, quando as peças são colocadas em uma câmara de pós-processo do próprio equipamento. O tempo da etapa de pós-cura foi de aproximadamente 24 minutos para cada peça fabricada.

Pode-se notar na Fig. 2 que a peça contém 9 formas geométricas repetidas, posicionadas em locais diferentes da plataforma para permitir a análise da influência da localização e a contração durante o processo de fabricação. Procura-se saber se a contração é mais severa no centro ou nas margens da plataforma de impressão. Foram atribuídos três níveis que correspondem às localizações norte $(+1)$, centro $(0)$ e sul $(-1)$ para o fator longitude, e as localizações oeste (+1), centro (0) e leste (-1) para o fator latitude.

A forma geométrica replicada na peça tem forma de cruz com as seguintes dimensões: $3,5 \mathrm{~mm}$ de comprimento em cada lado, largura de $0,6 \mathrm{~mm}$ e $0,8 \mathrm{~mm}$ de altura. A altura da cruz considerou a máxima amplitude de medida do perfilômetro digital Taylor Hobson modelo 112-2011, que é de $1 \mathrm{~mm}$. Foi utilizado um apalpador de $2 \mu \mathrm{m}$ de raio de ponta com ângulo de ponta de $45^{\circ}$. 
O formato de cruz permite a análise da contração em diferentes orientações no plano $x y$ do equipamento. Portanto, o comprimento do eixo da cruz orientado na direção $x$ é chamado de dimensão L $x$ da geometria, e seu ortogonal será chamado de dimensão Ly da geometria. Neste caso, procura-se saber se a dimensão $x$ ou $y$ da geometria pode ter correlação com as localizações da forma na plataforma. Cada aleta produzida pode ter duas posições: no eixo $x$, fator latitude, e no eixo y, que será chamado de fator longitude.
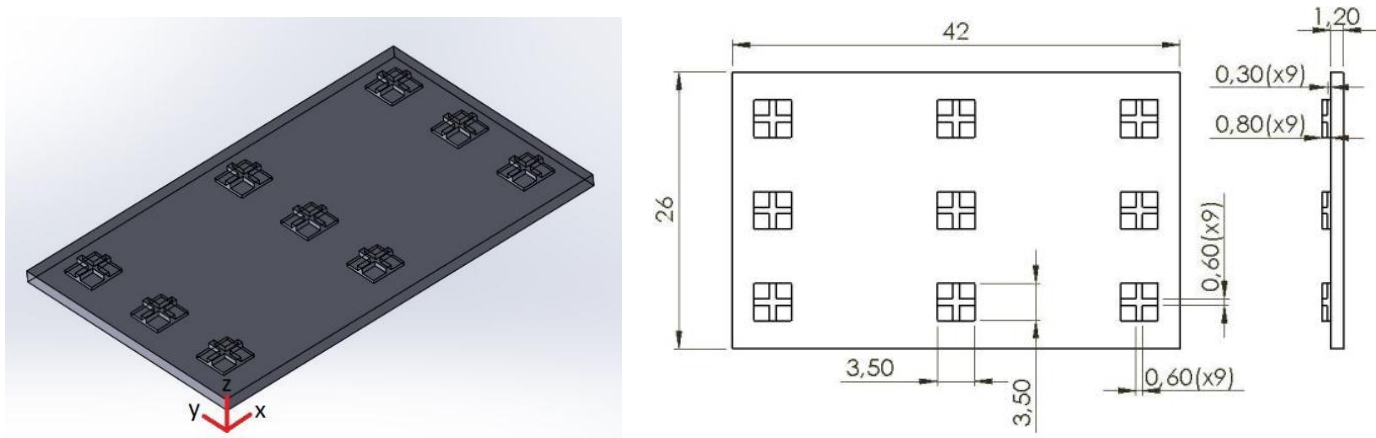

Figura 2: Vista isométrica e cotas do modelo digital da peça fabricada (valores em $\mathrm{mm}$ )

O fator de compensação de contração ajustado no equipamento foi mantido constante. Como a principal influência da taxa de sobre-cura é na direção de fabricação das camadas [13], a qual não foi analisada, este parâmetro foi mantido fixo na fabricação de todas as peças.

A Tabela 1 apresenta os fatores da localização de cada forma geométrica assim como seus níveis. As variáveis de saída analisadas foram as taxas de contração da dimensão $x, \mathrm{~S} x$ (\%), e da dimensão y, Sy (\%). Como o processo de fabricação foi realizado três vezes possuindo 2 fatores com 3 níveis cada um e duas variáveis de saída o número total de medições realizadas foi de 54.

A influência dos parâmetros na contração foi verificada a partir da análise de variância (ANOVA) de dois fatores, latitude e longitude, para a dimensão $x$ e $y$ da cruz. A interação dos fatores foi analisada para verificar se os fatores combinados apresentavam alguma influência significativa.

Tabela 1: Planejamento do Experimento

\begin{tabular}{c|c|c|c|c|c}
\hline FATORES & \multicolumn{3}{|c|}{ NÍVEIS } & \multicolumn{2}{c}{ SAÍDAS } \\
\hline Longitude & Norte (+1) & Centro (0) & Sul (-1) & S $x(\%)$ & Sy $(\%)$ \\
\hline Latitude & Oeste (+1) & Centro (0) & Leste (-1) & S $x(\%)$ & Sy $(\%)$ \\
\hline
\end{tabular}

\subsection{Metodologia para Medição do Perfil da Peça}

Após a pós-cura das peças, a topografia da peça foi medida com o perfilômetro. $\mathrm{O}$ perfil medido foi digitalizado para identificar as dimensões $\mathrm{L} x$ e Ly de cada forma. $\mathrm{O}$ arquivo digitalizado que representa $\mathrm{o}$ perfil medido é apresentado na Fig. 3.



Figura 3: Perfil da geometria da peça obtido pelo perfilômetro. 
Em seguida, os dados foram tratados na linguagem Matlab para obter a cota desejada nas direções $x$ e $y$, considerando a geometria do apalpador que limita a medida nos cantos vivos. A visualização do perfil corrigido pode ser vista na Fig. 4, onde as três retas indicam o perfil médio calculado a partir de uma regressão linear de cada região do perfil.

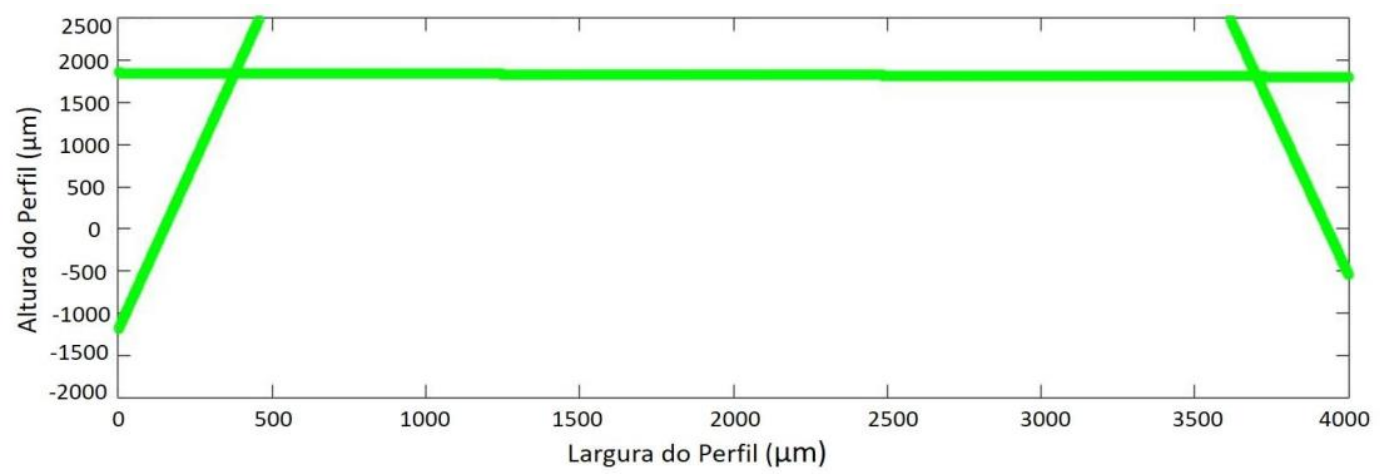

Figura 4: Perfil da geometria da peça corrigido com o algoritmo.

A contração $\mathrm{S}(\%)$ de cada dimensão da geometria foi calculada por meio da Eq. 1 a partir dos dados experimentais $\mathrm{L} i(\mathrm{~mm})$ na direção $i$ e o valor nominal $\mathrm{L} n(\mathrm{~mm})$ do modelo digital:

$S \%=\frac{L_{n}-L_{i}}{L_{n}} \times 100$

\section{RESULTADOS}

A partir dos valores de cada perfil geométrico com seu respectivo fator e nível, a porcentagem de contração foi calculada e com isso, foi realizada a análise de variância, a fim de avaliar o efeito de cada fator sobre esta alteração dimensional. Portanto, na seção seguinte são apresentados os resultados e na seção posterior são feitas as análises de variância.

\subsection{Dimensões e Contração}

Após a fabricação das peças não foi observada a deflexão da peça, fenômeno este reportado por Karalekas e Aggelopoulos [19], e que poderia influenciar a dimensão dos elementos. Os resultados medidos das dimensões $x$ e $y$ encontradas em cada elemento fabricado são mostrados na Tabela 2 e na Tabela 3, respectivamente, onde são apresentadas as contrações.

Para ilustrar a distribuição dos dados experimentais da contração para cada fator e seus respectivos níveis, dois gráficos em formato boxplot são demonstrados na Fig. 5 para direção x e na Fig. 6 para direção y.

Tabela 2: Dimensões Lx (mm) e Contrações Sx (\%) de cada elemento.

\begin{tabular}{|c|c|c|c|c|c|c|c|c|c|}
\hline \multirow{3}{*}{$\begin{array}{c}\text { Fator Longitude } \\
\mathrm{L} x(+1)\end{array}$} & \multicolumn{9}{|c|}{ Fator Latitude } \\
\hline & \multicolumn{3}{|c|}{ Oeste $(+1)$} & \multicolumn{3}{|c|}{ Centro (0) } & \multicolumn{3}{|c|}{ Leste (-1) } \\
\hline & 3,43 & 3,43 & 3,39 & 3,35 & 3,41 & 3,35 & 3,45 & 3,46 & 3,46 \\
\hline $\mathrm{S} x(\%)$ & 2,06 & 1,92 & 3,04 & 4,31 & 2,60 & 4,41 & 1,36 & 1,26 & 1,89 \\
\hline $\mathrm{L} x(0)$ & 3,39 & 3,45 & 3,36 & 3,40 & 3,38 & 3,36 & 3,43 & 3,45 & 3,33 \\
\hline $\mathrm{S} x(\%)$ & 3,29 & 1,37 & 3,98 & 2,93 & 3,44 & 4,07 & 2,04 & 1,36 & 4,77 \\
\hline $\mathrm{L} x(-1)$ & 3,39 & 3,45 & 3,42 & 3,37 & 3,39 & 3,36 & 3,42 & 3,43 & 3,44 \\
\hline $\mathrm{S} x(\%)$ & 3,03 & 1,44 & 2,15 & 3,81 & 3,18 & 3,96 & 2,20 & 1,91 & 1,64 \\
\hline
\end{tabular}

Tabela 3: Dimensões Ly (mm) e Contrações Sy (\%) de cada elemento. 


\begin{tabular}{c|c|c|c|c|c|c|c|c|c}
\hline & \multicolumn{9}{c}{ Fator Latitude } \\
\hline Fator Longitude & \multicolumn{3}{|c|}{ Oeste (+1) Centro (0) } & \multicolumn{3}{c}{ Leste (-1) } \\
\hline Ly (+1) & 3,37 & 3,36 & 3,37 & 3,35 & 3,42 & 3,34 & 3,38 & 3,40 & 3,39 \\
\hline Sy (\%) & 3,64 & 4,13 & 3,80 & 4,18 & 2,35 & 4,72 & 3,32 & 3,01 & 3,23 \\
\hline Ly (0) & 3,38 & 3,41 & 3,36 & 3,40 & 3,41 & 3,37 & 3,39 & 3,42 & 3,39 \\
\hline Sy (\%) & 3,31 & 2,69 & 4,00 & 2,82 & 2,37 & 3,84 & 3,25 & 2,29 & 3,09 \\
\hline Ly (-1) & 3,40 & 3,42 & 3,40 & 3,41 & 3,43 & 3,38 & 3,41 & 3,44 & 3,43 \\
\hline Sy (\%) & 2,85 & 2,22 & 2,90 & 2,54 & 1,98 & 3,55 & 2,54 & 1,62 & 1,88 \\
\hline
\end{tabular}
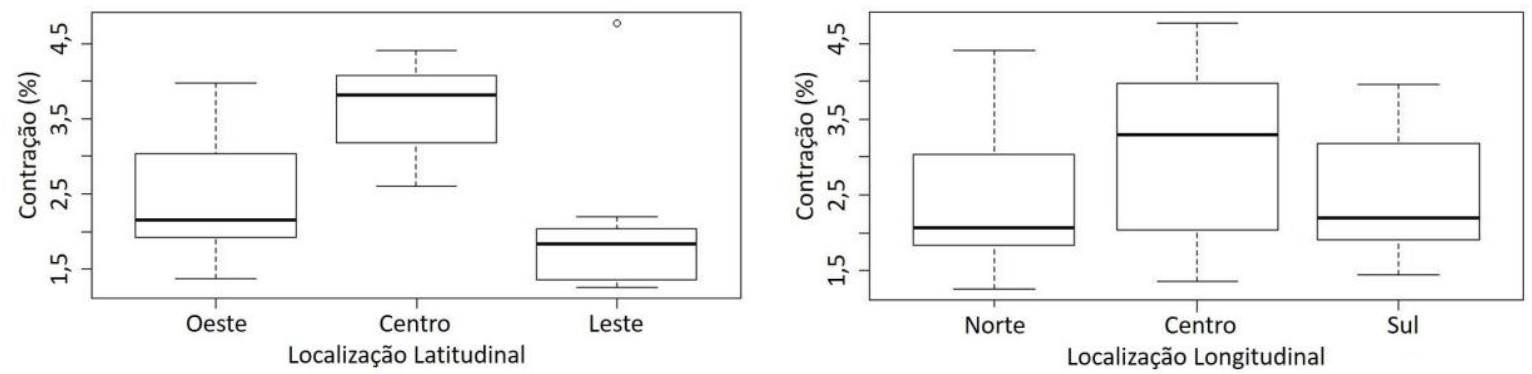

Figura 5: Boxplot dos resultados Sx.
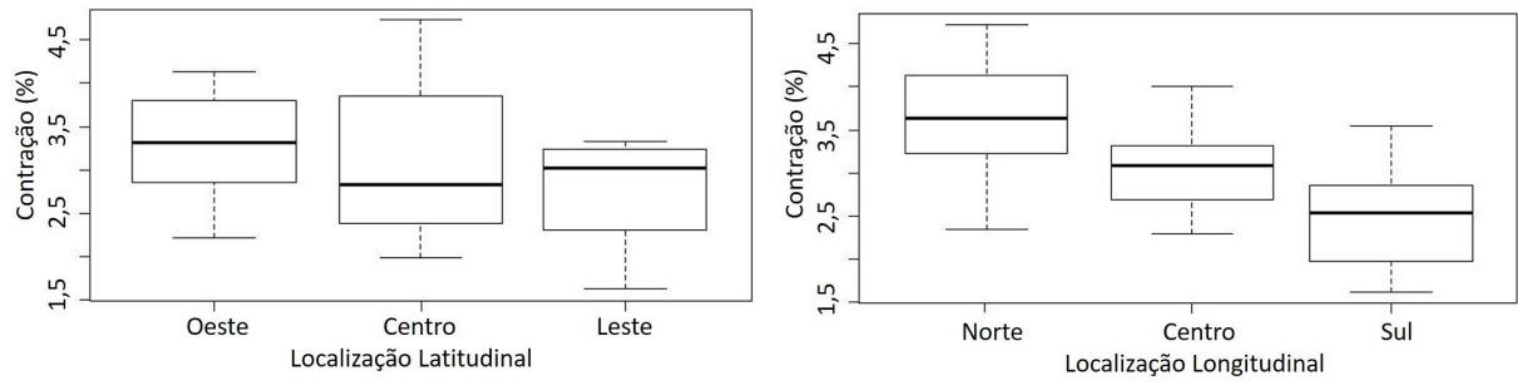

Figura 6: Boxplot dos resultados Sy.

\subsection{Análise de Variância}

Para cada orientação, foi realizada uma análise de variância com o auxílio do software R, incluindo os dois fatores e suas interações e os 3 níveis descritos. A Tabela 4 apresenta a tabela de ANOVA correspondente a Lx. Percebe-se que apenas o fator latitude é significativo, pois o valor p indica que, com 99,40\% de confiança, a localização latitudinal da peça na plataforma influencia a dimensão x.

Tabela 4: Tabela de ANOVA de dois fatores da dimensão $x$

\begin{tabular}{c|c|c|c|c|c}
\hline & $\begin{array}{c}\text { GRAUS DE } \\
\text { LIBERDADE }\end{array}$ & $\begin{array}{c}\text { SOMA DOS } \\
\text { QUADRADOS }\end{array}$ & $\begin{array}{c}\text { QUADRADOS } \\
\text { MÉDIOS }\end{array}$ & $\mathbf{F}$ & VALOR P \\
\hline LONGITUDE & 2 & 1,319 & 0,660 & 0,761 & 0,452 \\
\hline LATITUDE & 2 & 12,211 & 6,106 & 7,048 & 0,006 \\
\hline INTERAÇÃO & 4 & 1,950 & 0,487 & 0,563 & 0,693 \\
\hline ERRO & 18 & 15,593 & 0,866 & & \\
\hline TOTAL & 26 & 31,073 & & & \\
\hline
\end{tabular}

Sabendo que o fator latitude apresenta influência na contração de dimensões na direção do eixo x, pode-se perceber na distribuição vista na Fig. 5, que peças localizadas no centro latitudinal da plataforma tendem a apresentar maior contração na dimensão x. Neste ínterim, é possível perceber na mesma Fig. 5 que 
a localização longitudinal apresenta distribuições próximas umas das outras, confirmando a ANOVA que diz que o fator longitude não influencia na contração da dimensão x.

A partir da Tabela 5, é possível inferir que apenas o fator longitude é significativo para a dimensão y das geometrias, pois o valor p é baixo, levando a dizer com $99,40 \%$ de confiança que a localização longitudinal da peça na plataforma vai influenciar na dimensão y.

Tabela 5: Tabela de ANOVA de dois fatores para a dimensão y

\begin{tabular}{c|c|c|c|c|c}
\hline & $\begin{array}{c}\text { GRAUS DE } \\
\text { LIBERDADE }\end{array}$ & $\begin{array}{c}\text { SOMA DOS } \\
\text { QUADRADOS }\end{array}$ & $\begin{array}{c}\text { QUADRADOS } \\
\text { MÉDIOS }\end{array}$ & $\mathbf{F}$ & VALOR P \\
\hline LONGITUDE & 2 & 5,908 & 2,954 & 6,827 & 0,006 \\
\hline LATITUDE & 2 & 1,725 & 0,863 & 1,994 & 0,165 \\
\hline INTERAÇÃO & 4 & 0,256 & 0,064 & 0,148 & 0,962 \\
\hline ERRO & 18 & 7,787 & 0,433 & & \\
\hline TOTAL & 26 & 15,676 & & & \\
\hline
\end{tabular}

O fator longitude apresenta influência na contração de dimensões paralelas ao eixo y, e, portanto, pode-se perceber na Fig. 6 que peças localizadas no norte longitudinal da plataforma tendem a apresentar maior contração na dimensão y. Também na Fig. 6, fica evidente que a localização latitudinal tem suas distribuições próximas umas das outras, confirmando a ANOVA que diz que o fator latitude não influencia na contração da dimensão y.

Ou seja, no presente trabalho, a localização na plataforma é apresentada como fator de influência assim como observado por ZHOU et al. [16] em processos de SLA por escaneamento. No caso de SLA por escaneamento, cada camada é formada a partir de um feixe de laser que percorre a região da resina líquida que deve ser curada [12]. Devido a este tipo de configuração, o foco do laser no centro da plataforma é maior que nas partes mais externas, alterando assim o grau de contração das peças em função da sua posição na plataforma [20]. No entanto, no presente trabalho, foi utilizada a técnica de estereolitografia por projeção de radiação UV, sendo um mecanismo de formação diferente de cada camada, novos estudos devem ser realizados para melhor compreensão deste fenômeno.

\section{CONCLUSÕES}

Esse artigo abordou a influência que diferentes localizações das formas geométricas na plataforma e as variações nas dimensões x e y de uma peça fabricada por SLA por projeção. Para a análise da contração sob a influência da localização na plataforma, foi feita uma ANOVA de dois fatores, sendo esses fatores a localização latitudinal e longitudinal da cruz sobre a plataforma de impressão.

A partir da análise realizada, pode-se concluir que o fator latitude foi o único que apresentou influência sobre a contração na dimensão x e o fator longitude foi o único que apresentou influência sobre a contração na dimensão y. A interação entre os dois fatores não apresentou influência significativa sobre a contração das dimensões x e y. As dimensões x localizadas no centro da plataforma e as dimensões paralelas ao eixo y localizadas no norte da plataforma foram as que tiveram maior contração.

Há que se estudar as razões físicas que justifiquem os resultados experimentais da influência da posição na precisão das dimensões. Contudo, os resultados apresentados são relevantes, pois comprovam que a posição e a orientação das formas geométricas devem ser levadas em consideração para projetar dimensões que compensem a contração não homogênea em toda a plataforma, caso a precisão seja inferior à diferença de valores encontrados. Todos os valores de contração encontrados foram menores que 5\% para um elemento de $3 \mathrm{~mm}$ de comprimento.

\section{AGRADECIMENTOS}

Os autores agradecem o suporte financeiro do CNPq e aos recursos de aquisição do equipamento provenientes da emenda parlamentar do Tesouro Nacional apresentada pelo deputado federal Miro Teixeira. 


\section{BIBLIOGRAFIA}

[1] HULL, C. W., “Apparatus for Production of Three-Dimensional Objects by Stereolithography”, United States Patent 4575330, 11 Março 1986.

[2] LEAL, R., BARREIROS, F. M., ALVES, L., et al, "Additive manufacturing tooling for the automotive industry”, The International Journal of Advanced Manufacturing Technology, v. 92, pp 1671-1676, 2017.

[3] SEABRA, M., AZEVEDO, J, ARAÚJO, A, et al., "Selective laser melting (SLM) and topology optimization for lighter aerospace components”, Procedia Structural Integrity, v. 1, pp 289-296, 2016.

[4] TARAFDER, S., BOSE, S. "Polycaprolactone-Coated 3D Printed Tricalcium Phosphate Scaffolds for Bone Tissue Engineering: In Vitro Alendronate Release Behaviour and Local Delivery Effect on In Vivo Osteogenesis", ACS Applied Materials and Interfaces, v. 6, n. 13, pp. 9955-9965, 2014.

[5] DIKICI, B. A., DIKICI, S., KARAMAN, O., et al., "The effect of zinc oxide doping on mechanical and biological properties of 3D printed calcium sulfate based scaffolds", Biocybernetics and Biomedical Engineering, v. 37, pp 733-741, 2017.

[6] GUESSASMA, S., TAO, L., BELHABIB, S., et al., "Analysis of microstructure and mechanical performance of polymeric cellular structures designed using stereolithography”, European Polymer Journal, v. 98, pp 72-82, 2018.

[7] JUSKOVA, P., OLlitrAUlT, M., SERRA, M., et al., "Resolution improvement of 3D stereolithography through the direct laser trajectory programming: Application to microfluidic deterministic lateral displacement device", Analytica Chimica Acta, v. 1000, pp 239-247, 2018.

[8] LIFTON, V. A., LIFTON, G., SIMON, S., "Options for additive rapid prototyping methods (3D printing) in MEMS technology”, Rapid Prototyping Journal, v. 20, pp 403-412, 2014.

[9] MELChELS, F. P. W., FEIJEN, J., GRIJPMA, D. W., "A review on stereolithography and its applications in biomedical engineering”, Biomaterials, v. 31, pp 6121-6130, 2010.

[10] GUILlaumeA, O., GEVEN, M. A., SPRECHERA, C. M., et al., "Surface-enrichment with hydroxyapatite nanoparticles in stereolithography-fabricated composite polymer scaffolds promotes bone repair", Acta Biomaterialia, v. 54, pp 386-398, 2017.

[11] Rahmati S., Dickens, P., "Rapid tooling analysis of Stereolithography injection mould tooling" International Journal of Machine Tools and Manufacture, v. 47, pp 740-747, 2007.

[12] EMAMI, M.M., BARAZANDEH, F., YAGHMAIE, F., "Scanning-projection based stereolithography: Method and structure". Sensors and Actuators A: Physical, v. 218, pp. 116 - 124, 2014.

[13] COELHO, A.W.F., "Estudo do processo e da qualidade de peças fabricadas por estereolitografia”, Projeto Final de Curso de Graduação - Escola Politécnica - POLI/UFRJ, Rio de Janeiro, RJ, Brasil, 2016.

[14] JI, L., CHANG, W., CUI, M., et al., "Photopolymerization kinetics and volume shrinkage of 1,6hexanediol diacrylate at different temperature", Journal of Photochemistry and Photobiology A: Chemistry, v. 252, pp. 216-221, 2013.

[15] WANG, W., CHEAH, C., FUH, J., et al., "Influence of process parameters on stereolithography part shrinkage", Materials and Design, v. 17, n. 4, pp. 205-213, 1996.

[16] ZHOU, J.G., HERSCOVICI, D., CHEN, C.C., "Parametric process optimization to improve the accuracy of rapid prototyped stereolithography parts", International Journal of Machine Tools \& Manufacture, v. 40, pp. 363-379, 2000.

[17] HUANG, Q., ZHANG, J., SABBAGHI, A., et al., "Optimal offline compensation of shape shrinkage for three-dimensional printing processes”, IIE Transactions, v. 47, n. 5, pp. 431-441, 2017.

[18] LUAN, H., HUANG, Q., "Predictive modelling of in-plane geometric deviation for 3d printed freeform products", In: IEEE International Conference on Automation Science and Engineering (CASE), pp. 912-917, Gothenburg, Sweden, 2015.

[19] KARALEKAS, D., AGGEPOULOS, A., "Study of shrinkage strains in a stereolithography cured acrylic photopolymer resin”, Journal of Materials Processing Technology, v. 136, pp. 146-150, 2003.

[20] SAGER, B., ROSEN, D. W., SHILling, M., et al., "Experimental Studies in Stereolithography Resolution", In: Proceedings of the Solid Freeform Fabrication Symposium, pp. 70-81, Austin, Tex, USA, 2003. 